\title{
Edit Blindness: The relationship between attention and global change blindness in dynamic scenes.
}

\author{
Tim J. Smith \\ University of Edinburgh \\ John M. Henderson \\ University of Edinburgh
}

\begin{abstract}
Although we experience the visual world as a continuous, richly detailed space we often fail to notice large and significant changes. Such change blindness has been demonstrated for local object changes and changes to the visual form of whole images, however it is assumed that total changes from one image to another would be easily detected. Film editing presents such total changes several times a minute yet we rarely seem to be aware of them, a phenomenon we refer to here as edit blindness. This phenomenon has never been empirically demonstrated even though film editors believe they have at their disposal techniques that induce edit blindness, the Continuity Editing Rules. In the present study we tested the relationship between Continuity Editing Rules and edit blindness by instructing participants to detect edits while watching excerpts from feature films. Eye movements were recorded during the task. The results indicate that edits constructed according to the Continuity Editing Rules result in greater edit blindness than edits not adhering to the rules. A quarter of edits joining two viewpoints of the same scene were undetected and this increased to a third when the edit coincided with a sudden onset of motion. Some cuts may be missed due to suppression of the cut transients by coinciding with eyeblinks or saccadic eye movements but the majority seem to be due to inattentional blindness as viewers attend to the depicted narrative. In conclusion, this study presents the first empirical evidence of edit blindness and its relationship to natural attentional behaviour during dynamic scene viewing.
\end{abstract}

\section{Keywords: film editing, eye movement; real-world scene, naturalistic scene; oculomotor capture; gaze cue; continuity}

\section{INTRODUCTION}

Our experience of the visual world as a rich, highly detailed and continuous space is an illusion constructed from a series of momentary snapshots encoded while the eyes are still (fixations) and stitched together across periods of blindness as the eyes move (saccadic eye movements) (see Henderson, 2003, for summary). In order for us to maintain a representation of the scene across eye movements we must make predictions about the existence, location, and visual properties of objects not currently at the centre of our attention. The assumed continuity of these features make it possible for large and dramatic changes to go undetected (Change Blindness, see Simons and Levin, 1997) and unexpected and unattended features to go unnoticed (Inattentional Blindness, see Simons, 2000).

Change Blindness occurs when a visual change is masked by an artificial occlusion (Simons, 1996; Levin \& Simons, 1997; Rensink et al., 1997; Simons \& Levin, 1998; O’Regan et al., 1999; Rensink et al., 2000) or natural period of perceptual insensitivity, such as a saccadic eye movement or eye blink (Carlson-Radvansky \& Irwin, 1995; Grimes, 1996; Henderson \& Hollingworth, 1999; O'Regan et al., 2000), and participants fail to compare the changed scene to their memory of the scene (Brockmole \& Henderson, 2005; Hollingworth \& Henderson, 2002; Mitroff, Simons, \& Levin, 2004). Transsaccadic and long term memory studies have shown that relatively detailed information is 
retained across saccades and over time (Henderson \& Hollingworth, 1999; Hollingworth et al., 2001; Melcher, 2001; Hollingworth \& Henderson, 2002; Tatler, Gilchrist, \& Land, 2005; Hollingworth, 2006; also see Võ, Schneider, \& Matthias, and Humphrey \& Underwood in this volume) but changes to this information do not reach the level of awareness unless the change violates critical expectations about the continuity of the scene and objects within it (Levin \& Simons, 2000). The likelihood of violating these expectations and as a result detecting the change increases as the significance and size of a change within a scene increases (Rensink et al., 1997; Hollingworth \& Henderson, 2004; Levin \& Varakin, 2004; Henderson, Brockmole, \& Gajewski, 2008). Small local changes to object features (Henderson \& Hollingworth, 1999; Hollingworth et al., 2001; Hollingworth \& Henderson, 2002) and even slight global changes to the visual form of an entire scene (Henderson \& Hollingworth, 2003a; Hollingworth \& Henderson, 2004; Henderson, Brockmole, \& Gajewski, 2008) have been shown to sometimes go undetected, but it is assumed that a total change from one visual scene to a completely different visual scene that is not obscured in any way, e.g. by a mask, would be guaranteed to be detected.

Most of us experience total scene changes thousands of times every day in film and television edits, yet we seem to be unaware of most of them (Messaris, 1994). A typical ninety minute Hollywood film contains between one thousand and two thousand edits, a change in viewpoint ('shot') every 2.7 to 5.4 seconds (Bordwell \& Thompson, 2001), yet film editors assume that the majority of these edits are "invisible" as the "spectator's illusion of seeing a continuous piece of action is not interrupted" (Reisz \& Millar, 1953; pg 216). This assumption is prevalent throughout the filmmaking community but has never been empirically tested. If film viewers are unaware of some film edits - a phenomenon we call edit blindness - this would be evidence of extreme global change blindness. The main goal of this study was to see if there is any evidence that edit blindness occurs during the viewing of authentic feature films. If evidence of edit blindness can be found, the next step would then be to identify the conditions under which it occurs, how these relate to conventional editing practices, and what impact edit blindness has on our understanding of how we perceive a continuous visual world.
Although film has existed for over a century it has received very little psychological investigation. What little empirical research has been performed has typically investigated the relationship between the main structural conventions of Hollywood filmmaking, known as the Continuity Editing Rules, and our ability to comprehend narrative and spatial relationships (Frith \& Robson, 1975; Carroll \& Bever, 1976; Hochberg \& Brooks, 1978; Kraft, 1987; d'Ydewalle \& Vanderbeeken, 1990; Geiger \& Reeves, 1993; d'Ydewalle, Desmet, \& Van Rensbergen, 1998; Germeys \& d'Ydewalle, 2007). The Continuity Editing Rules are a suite of staging, filming, and editing conventions that a film maker can follow to ensure that the resulting film is not confusing, disorienting, or uncomfortable to watch (Reisz \& Millar, 1953; Bordwell \& Thompson, 2001; see Smith, 2006 for discussion). For example, one of the most prominent Continuity Editing Rules is the $180^{\circ}$ Rule (Bordwell \& Thompson, 2001, pages 263-265). This rule states that when filming a scene a main axis of action, down which most action occurs should be identified e.g. the line joining two characters engaged in a conversation. All static cameras used to film this scene should be positioned on the same side of this line. Keeping all cameras within the same $180^{\circ}$ arc ensures that when shots from these cameras are edited together the relative position of objects on the screen and direction of any action is consistent. It also ensures that the eyelines of multiple characters match even when the characters do not appear on screen at the same time. Cutting to a camera located on the opposite side of the axis of action (known as Crossing the Line) is believed to lead to confusion and disorientation ${ }^{1}$.

Film editors assume that one of the main benefits of adhering to the Continuity Editing Rules is edit blindness. This hypothesis has only been tested in two indirect empirical studies. Both studies asked participants to detect transitions from one viewpoint to another while watching films that either adhered to or violated the Continuity Editing Rules (d'Ydewalle \& Vanderbeeken, 1990; Schröder, 1990). Both studies found that cuts that violated the Continuity Editing Rules were detected faster than those that adhered to the rules, indicating more awareness of the discontinuity cuts (d'Ydewalle \&

\footnotetext{
${ }^{1}$ For empirical investigations of the impact of crossing the line see Frith \& Robson (1975), Kraft (1987), d'Ydewalle, Desmet, \& Van Rensbergen (1998), and Germeys $\&$ d'Ydewalle (2007).
} 
Vanderbeeken, 1990; Schröder, 1990). However, these studies do no speak to the question of whether participants were aware of continuity cuts. In order to investigate this issue we first need to understand the conditions under which film editors believe edit blindness may occur.

Only a few film theorists have questioned how the Continuity Editing Rules create edit blindness. These theorists have provided a range of intriguing hypotheses that, on examination, may relate Continuity Editing directly to Inattentional Blindness and Change Blindness. Editors believe awareness of film editing can be minimised by coinciding cuts with moments when attention is occupied by another task such as comprehending the narrative (Reisz \& Millar, 1953; Bordwell \& Thompson, 2001), or hiding the cut transients during sudden onsets of motion (Reisz \& Millar, 1953; Dmytryk, 1986; Katz, 1991; Anderson, 1996; Bordwell \& Thompson, 2001; Pepperman, 2004), saccadic eye movements (Dmytryk, 1986), or eye blinks (Dmytryk, 1986; Murch, 2001). These techniques parallel the methods used in Change Blindness studies to obscure the visual transients associated with a change (CarlsonRadvansky \& Irwin, 1995; Grimes, 1996; Henderson \& Hollingworth, 1999; O’Regan et al., 1999; Rensink et al., 2000; O’Regan et al., 2000).

Both eye blinks and saccadic eye movements provide a period of perceptual insensitivity due to neural suppression (Bristow et al., 2005; Diamond, Ross, \& Morrone, 2000). Eye blinks occur 10-15 times a minute (once every 4-6 seconds) and last 100-150ms with the period of perceptual insensitivity extending for a few extra milliseconds before and after the blink itself (Burr, 2005). Saccadic eye movements occur more frequently, 2-5 times per second (once every 200-500ms; Yarbus, 1967), last less time $(20-50 \mathrm{~ms})$, but have a period of perceptual insensitivity similar in duration to blinks, around $75 \mathrm{~ms}$ before and $50 \mathrm{~ms}$ after the eye movement (Diamond, Ross, \& Morrone, 2000). The relative infrequency of blinks and the short duration of saccadic eye movements would mean that deliberately using these periods of perceptual insensitivity to hide cuts would require very precise timing. Such timing may be provided by the Continuity Editing Rules.

The most important Continuity Editing technique, to which most other rules are applied, is known as the analytical breakdown of scenes or Within Scene editing
(Reisz \& Millar, 1953; Bordwell \& Thompson, 2001). Presenting a scene as a series of shots, beginning with the camera further away from the action (Long Shots) and gradually moving closer (Medium Shots and Close-Ups; see Figure 1, Within Scenes) adds interest to the presentation of the scene and provides the viewer with the optimum viewpoint on the action (Hochberg \& Brooks, 1978). Given that a film viewer's primary interest is following the film's narrative, engaging them in this task by constantly changing viewpoint within a scene is believed to occupy attention and limit awareness of the editing (Reisz \& Millar, 1953; Bordwell \& Thompson, 2001). The technique of focusing attention on one task, e.g. instructing viewers to count the number of passes during a basketball game, in order to limit awareness of unrelated visual features, e.g. a man in a Gorilla suit, is commonly referred to as Inattentional Blindness (Mack \& Rock, 1998; Simons \& Chabris, 1999; Simons, 2000). Within Scene editing may utilise the Inattentional Blindness created by focussing attention on the narratively significant action in order to limit the resources available for noticing the editing. However, Inattentional Blindess is typically only reported for local features within a scene (Mack \& Rock, 1998; Simons \& Chabris, 1999). Edit Blindness following Within Scene edits would be the first example of Inattentional Blindness for total global scene changes.

Inducing inattentional blindness via Within Scene editing may not be sufficient to minimize awareness of the visual transients associated with the cut. As in Change Blindness, the transients may need to be obscured, occluded, or suppressed. According to film editors, two sub-types of Within Scene edits may provide such periods of insensitivity: Match Action and Gaze Match edits. The Match Action editing rule (see Figure 1, third row) states that a smooth cut between two viewpoints of the same action will be achieved by coinciding the cut with a sudden onset of motion (Reisz \& Millar, 1953; Dmytryk, 1986; Katz, 1991; Anderson, 1996; Bordwell \& Thompson, 2001; Pepperman, 2004). This technique may function by either obscuring the cut with motion blur (Pepperman, 2001), creating a saccadic eye movement towards the motion (Dmytryk, 1986) or, with extreme changes in action, causing an eye blink (Dmytryk, 1986; Murch, 2001).

Saccadic suppression of the cut transients is also thought to be used by Gaze Match editing to limit 
awareness of cuts. The Gaze Match editing rule (see Figure 1, fourth row) suggests that cutting from a shot of an actor looking off-screen to a shot of the target of the actor's gaze will result in a seamless transition between the shots (Reisz \& Millar, 1953). This technique is believed to result in a saccadic eye movement along the line of the actor's gaze in anticipation of the cut (Dmytryk, 1986). Such attentional cuing by gaze shifts has been demonstrated using abstract and isolated human faces (Friesen \& Kingstone, 1998; Langton, Watt \& Bruce, 2000), realistic faces in static photographs (Castelhano, Wieth, \& Henderson, 2007; Birmingham, Biscof, \& Kingstone, 2007) and has also been observed in dynamic scenes (Kuhn \& Tatler, 2005; Tatler \& Kuhn, 2007). However, in order for gaze cues to be used reliably by editors to create edit blindness the majority of viewers would have to respond to the cue at the same time and the Continuity Editing rules would have to identify the precise timing of this response. Whether both of these requirements exist will be investigated in this study.

The present study investigated the existence of edit blindness by instructing participants to detect edits while watching excerpts from seven feature films. Eye movements were recorded during the task in order to identify whether undetected cuts coincided with periods of perceptual insensitivity such as saccades or blinks as hypothesized by film editors. All edits were categorised according to their adherence to the Continuity Editing Rules: Between Scenes (no continuity), Within Scenes (scene continuity), Match Action (scene and action continuity), and Gaze Match (scene and gaze continuity). The four edit types are illustrated in Figure 1. If the application of the Continuity Editing techniques results in edit blindness, A) participants should be less likely to detect cuts that utilise these techniques compared with cuts that do not, i.e. Between Scene, B) detection of these cuts should be slower, and $C$ ) there should be evidence that the cuts that are not detected utilise one or more of the techniques for obscuring the transients of the cut outlined above: coincidence with motion blur, saccadic eye movement, or eye blink. Support for these hypotheses would provide the first empirical evidence of edit blindness during film viewing.

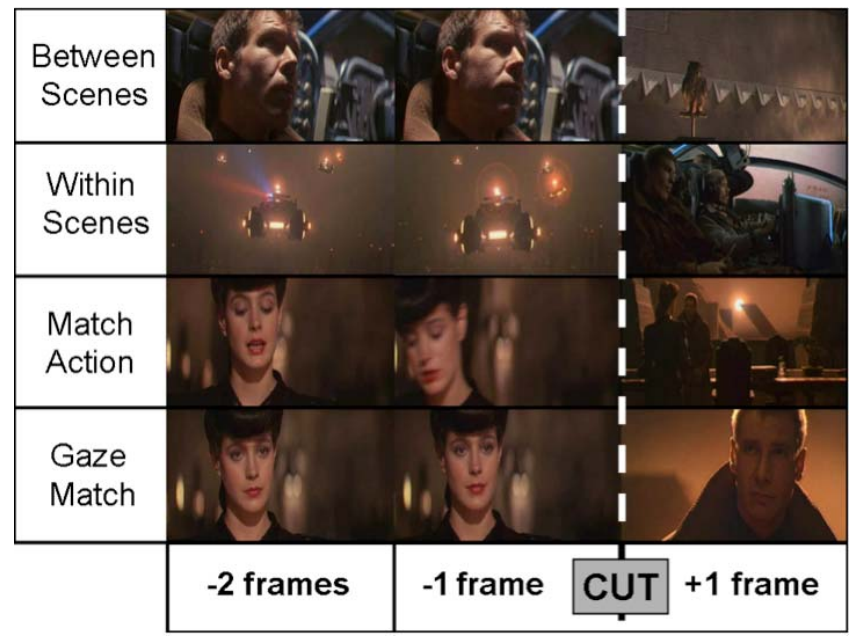

Figure 1: Examples of the four categories of cuts compared in this study: Between Scenes (no continuity; top row), Within Scenes (scene continuity; second row), Match Action (scene and action continuity; third row), and Gaze Match (scene and gaze continuity; bottom row). All images are taken from Blade Runner (Ridley Scott, 1982) and depict the two frames prior to the cut and the frame immediately after the cut.

\section{METHOD}

\section{Participants.}

Seven members of the Edinburgh University community ( 3 female; mean age $=28.7$ years) participated for payment (£5). All participants had normal or corrected to normal vision. Participation was voluntary. The experiment was conducted according to the British Psychological Society's ethics guidelines.

\section{Apparatus.}

Eye movements were monitored by an SR Research Eyelink II eyetracker sampling eye position at $500 \mathrm{~Hz}$. Viewing was binocular, but only the right eye was tracked. The images were presented on a 21 inch cathode ray tube (CRT) monitor at a viewing distance of around $60 \mathrm{~cm}$ (participants were free to move their head ${ }^{2}$ ) with a refresh rate of $100 \mathrm{~Hz}$, resolution of $800 \times 600$ pixels $x$ 32 bit. The experiment was controlled with SR Research Experiment Builder software. Responses were made via a

\footnotetext{
${ }^{2}$ Participants mostly kept their heads stationary during each trial and any minor head movements were automatically compensated for by the eye tracker without impairing tracker accuracy.
} 
Microsoft Sidewinder joypad. Raw gaze data and responses were time-locked to the video frames and recorded to the hard disk after the experiment. The analysis utilised both the raw and filtered data using SR Research Data Viewer to identify saccades, fixations and eye blinks. Saccades were detected using a 9-sample saccade detection model with a velocity threshold of $30 \% \mathrm{sec}$, an acceleration threshold of $8000^{\circ} / \mathrm{sec}^{2}$, and a minimum amplitude of $0.5^{\circ}$. Eye blinks were identified whenever the pupil was occluded, e.g. by the eyelid, lost or severely distorted. Analysis of the eye movement data was performed via Matlab and SPSS.

\section{Stimuli.}

Participants were presented seven five-minute continuous excerpts extracted from feature films: Blade Runner (Ridley Scott, 1982), Citizen Kane (Orson Welles, 1941), Dogville (Lars Von Trier, 2003), October (Sergei Eisenstein, 1928), Requiem for a Dream (Darren Aronofsky, 2000), Dancer in the Dark (Lars Von Trier, 2000), and Koyaanisqatsi (Godfrey Reggio, 1982). These films were chosen as they covered a cross-section of film making styles and practices and vary in the degree to which they adhere to the continuity editing rules. For example, Blade Runner (Ridley Scott, 1982) utilises very strict and classic continuity while Dancer in the Dark (Lars Von Trier, 2000) consciously violates the continuity editing rules.

Excerpts from these films were chosen according to the following constraints. Each excerpt must begin at the start of a scene, not contain titles or subtitles ${ }^{3}$, last for exactly five minutes, and not contain offensive or violent imagery. Excerpts were extracted from digital versions of each film and rendered in the XviD MPEG-4 digital video format at a resolution of 720x576 and 25fps (DVD quality). Stereo audio was also extracted and synchronised to the video as a Wave file. These formats were chosen as they ensure synchronisation of the gaze recording and the video playback through Experiment Builder. The order in which the videos were presented was randomised across participants.

\footnotetext{
${ }^{3}$ An exception was made for October (Sergei Eisenstein, 1928) a silent film using textual descriptions (intertitles) inserted between shots to communicate the narrative. All cuts to and from intertitles were excluded in the subsequent analysis.
}

\section{Procedure.}

Participants were told that they would be shown seven clips from feature films. Each clip would last for five minutes. Their task was to watch the videos and press a button every time they saw an edit. An edit was defined as "Any transition from one viewpoint to another that could not have been produced by a single continuously filming camera." A broad definition of 'edit' was used to ensure that participants would also identify optical transitions such as fades and wipes and digital effects such as the transformation of one image into another as edits. Participants were instructed that their responses would be timed so they should respond as quickly as possible. Participant understanding of the edit detection task was tested using a 2 minute practice film.

The Eyelink II head-mounted eye tracker was securely but comfortably fastened on the participants head. A latex swimming cap was worn over their hair to minimise slippage. The head position and distance relative to the monitor was checked and the right eyetracker camera was positioned. The main experiment began with a nine-point calibration of the eyetracker. Calibration was deemed acceptable if the gaze position was within $0.5^{\circ}$ of a visual angle of the target. Each video was presented without interruption while the participant detected edits by pressing a button on the joypad. After each video a further drift correction was performed to compensate for drift and slippage.

After all seven videos had been presented participants completed a short questionnaire about the films. The questionnaire checked whether they had previously seen the films and how familiar the films were. All participants stated that they had either never seen the films before or could not recall them in detail. The whole experiment lasted less than 45 minutes.

\section{Coding and Analysis.}

In order to identify the impact of the continuity editing rules on participant awareness of film edits, all edits in the sample films were hand coded for their adherence to the rules. The films were coded according to five categories:

1) Edit Type = Cut, Other. Was the transition between the two shots a straight cut? 


\begin{tabular}{|l|c|c|c|c|c|c|c|c|}
\hline Film & $\begin{array}{l}\text { Num. } \\
\text { Edits }\end{array}$ & $\begin{array}{c}\text { ASL } \\
(\mathbf{s e c})\end{array}$ & $\begin{array}{l}\text { Cut } \\
(\%)\end{array}$ & $\begin{array}{l}\text { Size } \\
(\%)\end{array}$ & $\begin{array}{c}\text { Between } \\
\text { Scene } \\
(\%)\end{array}$ & $\begin{array}{c}\text { Within } \\
\text { Scene } \\
(\%)\end{array}$ & $\begin{array}{c}\text { Match } \\
\text { Action } \\
(\%)\end{array}$ & $\begin{array}{c}\text { Gaze } \\
\text { Match } \\
(\%)\end{array}$ \\
\hline Blade Runner & 74 & 4.1 & 100 & 74 & 6 & 16 & 0 & 49 \\
\hline Citizen Kane & 47 & 6.4 & 96 & 53 & 13 & 4 & 4 & 17 \\
\hline Dogville & 47 & 6.4 & 100 & 55 & 0 & 15 & 15 & 15 \\
\hline October & 111 & 2.7 & 55 & 34 & 25 & 3 & 8 & 3 \\
\hline Requiem for a Dream & 108 & 2.8 & 100 & 59 & 9 & 20 & 13 & 3 \\
\hline Dancer in the Dark & 49 & 6.1 & 100 & 82 & 18 & 13 & 0 & 10 \\
\hline Koyaanisqatsi & 20 & 15 & 95 & 26 & 40 & 20 & 0 & 0 \\
\hline All & $\mathbf{6 5 . 1}$ & $\mathbf{4 . 6}$ & $\mathbf{9 2}$ & $\mathbf{5 9 . 7}$ & $\mathbf{1 2 . 2}$ & $\mathbf{1 3 . 4}$ & $\mathbf{6 . 3}$ & $\mathbf{1 6 . 5}$ \\
\hline
\end{tabular}

Table 1: General edit statistics for the seven feature film excerpts used in this study: Number of edits in the 5 minute excerpt (Num. Edits), Average Shot Length (ASL; seconds), Percentage of edits that are cuts (Cut; \%), Percentage of cuts that were of shot size (Size; \%) Close-Up (CU), Close Medium Shot (CMS), orMedium Shot (MS), Percentage of cuts of size CU, CMS, or MS that were classified as Between Scene, Within Scene, Match Action, or Gaze Match (\%). Averages across all seven films are displayed in the bottom row.

2) Shot Size = eXtreme Close-Up (XCU), CloseUp (CU), Close Medium Shot (CMS), Medium Shot (MS), Medium Long Shot (MLS), Long Shot (LS), eXtreme Long Shot (XLS). All shot sizes are specified relative to how much of a human figure would be visible in the frame if the top of the head was touching the top of the screen (Katz, 2001). A CU shows an entire head, MS shows the upper torso and LS a full body. The shot size following the cut is coded.

\section{3) Scene Continuity = Within Scene}

(Continuity), Between Scene (Discontinuity).

Two shots are coded as belonging to the same scene if a focal object e.g. a person, belonging to the current scene is immediately present in the new shot.

4) Match on Action = Yes, No. Two shots are coded as being joined by a Match Action edit if the subject of both shots is the same e.g. a person or object, and the subject begins an action in the first shot and continues it in the second shot.
5) Gaze Match = Yes, No. Two shots are coded as being joined by a Gaze Match edit if the shots depict different subjects and both are looking offscreen in the direction of the other person, i.e. if the two images were overlaid their eyelines would meet. Cuts involving eyelines that do not meet or in which the screen location of the two people are identical across the cut were excluded.

Coding was performed by two independent coders both following the same guidelines. At the beginning of every shot the coder identified the size of the shot (Shot Size), whether the edit preceding the shot was a cut (Edit Type), and whether the two shots either side of the edit had continuity of scene (Within Scene), action (Match Action), or gaze (Gaze Match). Intercoder reliability across the five categories was on average $85 \%$. 


\begin{tabular}{|c|c|c|c|c|c|c|c|c|c|c|}
\hline & \multicolumn{5}{|c|}{ Edits Missed (\%) } & \multicolumn{5}{|c|}{ Reaction Time (ms) } \\
\hline Film & All & BS & WS & MA & GM & All & BS & WS & MA & GM \\
\hline Blade Runner & $\begin{array}{c}8.6 \\
(8.4)\end{array}$ & $\begin{array}{l}19.1 \\
(18)\end{array}$ & $\begin{array}{c}4.8 \\
(8.7)\end{array}$ & - & $\begin{array}{c}9.0 \\
(9.5)\end{array}$ & $\begin{array}{l}353 \\
(67)\end{array}$ & $\begin{array}{c}420 \\
(159)\end{array}$ & $\begin{array}{c}289 \\
(176)\end{array}$ & - & $\begin{array}{l}351 \\
(66)\end{array}$ \\
\hline Citizen Kane & $\begin{array}{c}9.2 \\
(10)\end{array}$ & $\begin{array}{c}4.8 \\
(13)\end{array}$ & $\begin{array}{l}14.3 \\
(38)\end{array}$ & $\begin{array}{l}14.3 \\
\text { (38) }\end{array}$ & $\begin{array}{c}3.7 \\
(9.4)\end{array}$ & $\begin{array}{l}569 \\
(62)\end{array}$ & $\begin{array}{c}623 \\
(125)\end{array}$ & $\begin{array}{c}550 \\
(103)\end{array}$ & $\begin{array}{c}526 \\
(177)\end{array}$ & $\begin{array}{c}508 \\
(140)\end{array}$ \\
\hline Dogville & $\begin{array}{l}15.0 \\
(13)\end{array}$ & - & $\begin{array}{l}39.3 \\
(34)\end{array}$ & $\begin{array}{l}10.7 \\
(20)\end{array}$ & $\begin{array}{l}21.4 \\
(22)\end{array}$ & $\begin{array}{l}603 \\
(86)\end{array}$ & - & $\begin{array}{c}680 \\
(114)\end{array}$ & $\begin{array}{c}605 \\
(121)\end{array}$ & $\begin{array}{c}577 \\
(129)\end{array}$ \\
\hline October & $\begin{array}{c}7.7 \\
(4.2)\end{array}$ & $\begin{array}{c}0 \\
(0)\end{array}$ & $\begin{array}{c}0 \\
(0)\end{array}$ & $\begin{array}{l}29.6 \\
(38)\end{array}$ & $\begin{array}{c}0 \\
(0)\end{array}$ & $\begin{array}{l}466 \\
(46)\end{array}$ & $\begin{array}{l}430 \\
(60)\end{array}$ & $\begin{array}{c}434 \\
(104)\end{array}$ & $\begin{array}{c}659 \\
(480)\end{array}$ & $\begin{array}{l}428 \\
(74)\end{array}$ \\
\hline Requiem for a Dream & $\begin{array}{l}30.0 \\
(32)\end{array}$ & $\begin{array}{l}20.5 \\
(38)\end{array}$ & $\begin{array}{l}37.5 \\
(31)\end{array}$ & $\begin{array}{l}47.3 \\
(31)\end{array}$ & $\begin{array}{l}28.6 \\
\text { (53) }\end{array}$ & $\begin{array}{l}452 \\
(74)\end{array}$ & $\begin{array}{c}490 \\
(145)\end{array}$ & $\begin{array}{l}437 \\
(89) \\
\end{array}$ & $\begin{array}{l}486 \\
(83) \\
\end{array}$ & $\begin{array}{c}507 \\
(134)\end{array}$ \\
\hline Dancer in the Dark & $\begin{array}{l}14.3 \\
(9.1) \\
\end{array}$ & $\begin{array}{l}14.3 \\
(12) \\
\end{array}$ & $\begin{array}{l}20.0 \\
(16) \\
\end{array}$ & - & $\begin{array}{l}14.3 \\
(20) \\
\end{array}$ & $\begin{array}{l}578 \\
(73)\end{array}$ & $\begin{array}{l}578 \\
(50)\end{array}$ & $\begin{array}{c}608 \\
(108) \\
\end{array}$ & - & $\begin{array}{l}560 \\
(94)\end{array}$ \\
\hline Koyaanisqatsi & $\begin{array}{c}7.1 \\
(5.4)\end{array}$ & $\begin{array}{c}0 \\
(0)\end{array}$ & $\begin{array}{c}57 \\
(53)\end{array}$ & & & $\begin{array}{c}643 \\
(319)\end{array}$ & $\begin{array}{c}618 \\
(274)\end{array}$ & $\begin{array}{c}635 \\
(124)\end{array}$ & - & \\
\hline All & $\begin{array}{l}15.8 \\
(10)\end{array}$ & $\begin{array}{c}9.4 \\
(8.4)\end{array}$ & $\begin{array}{l}25.1 \\
(11)\end{array}$ & $\begin{array}{l}32.4 \\
(14)\end{array}$ & $\begin{array}{l}10.9 \\
(8.4)\end{array}$ & $\begin{array}{l}489 \\
(53)\end{array}$ & $\begin{array}{l}507 \\
(59)\end{array}$ & $\begin{array}{c}451 \\
(116)\end{array}$ & $\begin{array}{l}564 \\
(79)\end{array}$ & $\begin{array}{c}410 \\
(55)\end{array}$ \\
\hline
\end{tabular}

Table 2: Percentage of edits missed (\%) and reaction times (ms) for detected edits. Results are split by film (rows), presented for all edits (vertical light grey, 'All' columns) and a subset of edits that are cuts with shot size CU, CMS, or MS and type Between Scene (BS), Within Scene (WS), Match Action (MA), and Gaze Match (GM). Means across all films are presented on the bottom row. Standard Deviations are presented in parantheses

\section{RESULTS}

\section{Percentage of Edits Missed}

The first and most important measurement for identifying whether edit blindness occurred was the percentage of edits that were not detected. Overall $15.8 \%$ of all edits were missed (see Table $2,1^{\text {st }}$ block). As can be seen from Table 2, the Percentage of Edits missed varied considerably across the seven films. These differences could be driven by a number of factors such as shot content, edit type, shot length, etc. To test the specific hypotheses about the Continuity Edits such potential confounding factors need to be excluded. Only edits involving straight cuts, i.e. instantaneous transitions between shots, not gradual effects such as dissolves or fades, were used. In order to minimise confounds of shot content the cuts used in the analysis had to be cuts to shots of size Close-Up (CU), Close Medium Shot (CMS), or Medium Shot (MS). This ensured that all cuts were followed by similarly composed visual scenes with similar contents e.g. at least one person. From the cuts meeting these requirements four sub-classes were identified according to the cuts adherence to the Continuity Editing Rules: Between Scene (no continuity), Within Scene (scene continuity), Match Action (scene and action continuity), and Gaze Match (scene and gaze continuity). The edits that satisfied all these requirements are referred to as valid cuts. All other types of edits were excluded from the analysis. 117 cuts remained after all exclusions: Between Scene = 26, Within Scene $=34$, Match Action = 15, and Gaze match $=42$.

Repeated-measures ANOVA for the percentage of cuts missed across the four cut types (Table 2, bottom row, $1^{\text {st }}$ block) indicates a main effect of Cut Type $(F(3,18)=29.970, p<.001)^{4}$. This can be attributed to Within Scene and Match Action cuts which both had

\footnotetext{
${ }^{4}$ Film could not be included as a factor as the four cut types were not represented across all films.
} 
significantly greater number of cuts missed $(25.1 \%$ and $32.4 \%$, respectively) compared with Between Scene (9.4\%; both $\mathrm{p}<.01)^{5}$ and Gaze Match (10.9\%; both $\mathrm{p}<.05)$. There were no significant differences between the percentage of cuts missed for Gaze Match and Between Scene cuts or Within Scene and Match Action cuts. This supports our hypothesis that scene (Within Scene) and action continuity (Match Action) increase edit blindness relative to no continuity (Between Scene).. However, there is no indication that gaze continuity (Gaze Match) increased edit blindness.

\section{Time taken to detect cuts.}

Across all films and cut types the average cut detection time was $489 \mathrm{~ms}$ (see Table $2,2^{\text {nd }}$ block). This varied considerably across films with Blade Runner, one of the films expected to exhibit a high degree of continuity, demonstrating the quickest cut detection time (353ms) where as Koyaanisqatsi, the film with the lowest percentage of missed cuts having the slowest detection time $(643 \mathrm{~ms})$.

A repeated-measures ANOVA for cut detection times across the four cut types (Table 2, bottom row, $2^{\text {nd }}$ block) indicated a main effect for Cut Type $(\mathrm{F}(3,18)=6.320, \quad \mathrm{p}<.01)$. This difference can be attributed to Gaze Match cuts being detected significantly faster $(410 \mathrm{~ms})$ than Between Scene $\left(507 \mathrm{~ms} ; \mathrm{p}<.05^{4}\right)$ and Match Action cuts $(564 \mathrm{~ms}$; $\mathrm{p}<.01)$. There were no other significant differences. These results do not support our hypotheses that Within Scene, Match Action, or Gaze Match cuts delay detection compared with Between Scene cuts but there is a non significant trend in this direction for Match Action cuts ( $\mathrm{p}=.127$, n.s.). A positive correlation between reaction time and percentage of cuts missed was also predicted by our hypotheses but no significant correlation is found: Between Scene $\left(\chi^{2}=0.281, \mathrm{~N}=7\right.$, $\mathrm{p}=.54$, n.s. $)$ Within Scene $\left(\chi^{2}=-0.327, \mathrm{~N}=7, \mathrm{p}=.47\right.$, n.s.), Match Action $\left(\chi^{2}=-0.435, \mathrm{~N}=7, \mathrm{p}=.33\right.$, n.s. $)$, and Gaze Match $\left(\chi^{2}=0.185, \mathrm{~N}=7, \mathrm{p}=.69\right.$, n.s. $)$. There is also no significant correlation across all types of cuts $\left(\chi^{2}=0.097, \mathrm{~N}=147, \mathrm{p}=.24\right.$, n.s. $)$.

The results from the cut detection task presented above indicate that Match Action and Within Scene

\footnotetext{
${ }^{5}$ All post-hoc comparisons are performed with Bonferroni correction for multiple comparisons.
}

cuts produce more edit blindness than Between Scene and Gaze Match cuts. This establishes that edit blindness does exist and seems to be related to the application of certain Continuity Editing rules. The next stage in this analysis is to test whether there is a higher incidence of saccadic eye movements or eye blinks during these missed cuts. A significantly higher probability of saccades or blinks during missed cuts compared with detected cuts may suggest a mechanism by which the transients associated with some cuts were suppressed, limiting viewer awareness of the editing.

\section{Coincidence of blinks and cuts.}

Across all edits the probability that a blink occurred at the exact moment of an edit was very low (1.2\%.) The percentage of detected valid cuts that coincided with an eye blink was $1.09 \%$ and for missed cuts, $1.06 \%$. Across the seven films the percentage of missed cuts that coincided with eye blinks was negligible: Blade Runner $=2.3 \%$, Citizen Kane $=$ $4.8 \%$, Dogville $=0 \%$, October $=0 \%$, Requiem for a Dream $=0.93 \%$, Dancer in the Dark $=0 \%$, and Koyaanisqatsi $=0 \%$. When the data were collapsed across all films and split by the four cut types none of the cut types had any missed cuts coinciding with blinks.

In order to account for the period of perceptual insensitivity beginning a few milliseconds before and extending a few milliseconds after the period of the blink itself (Burr, 2005) the probability of a blink occurring $100 \mathrm{~ms}$ before and after the cut was calculated. This extended time window still revealed no blinks for missed cuts across most edit types. However, $11.6 \%$ of missed Within Scene cuts exhibited a blink in this time window compared with only $3.3 \%$ of detected cuts. A paired-samples t-test between the blink probability for detected and undetected Within Scene cuts revealed a marginally significant difference, $\mathrm{t}(6)=-2.426, \mathrm{p}=.051$. This indicates that a small proportion $(11.6 \%)$ of the Within Scene cuts that failed to be detected coincided with a period of perceptual sensitivity that may have limited awareness of the visual transients associated with the cut. For these cuts, a participant would have had to perform overt comparison of the new shot to their memory of the old shot in order to detect the cut. No such coincidence with blinks was observed for Between Scene, Match Action, or Gaze Match cuts. 


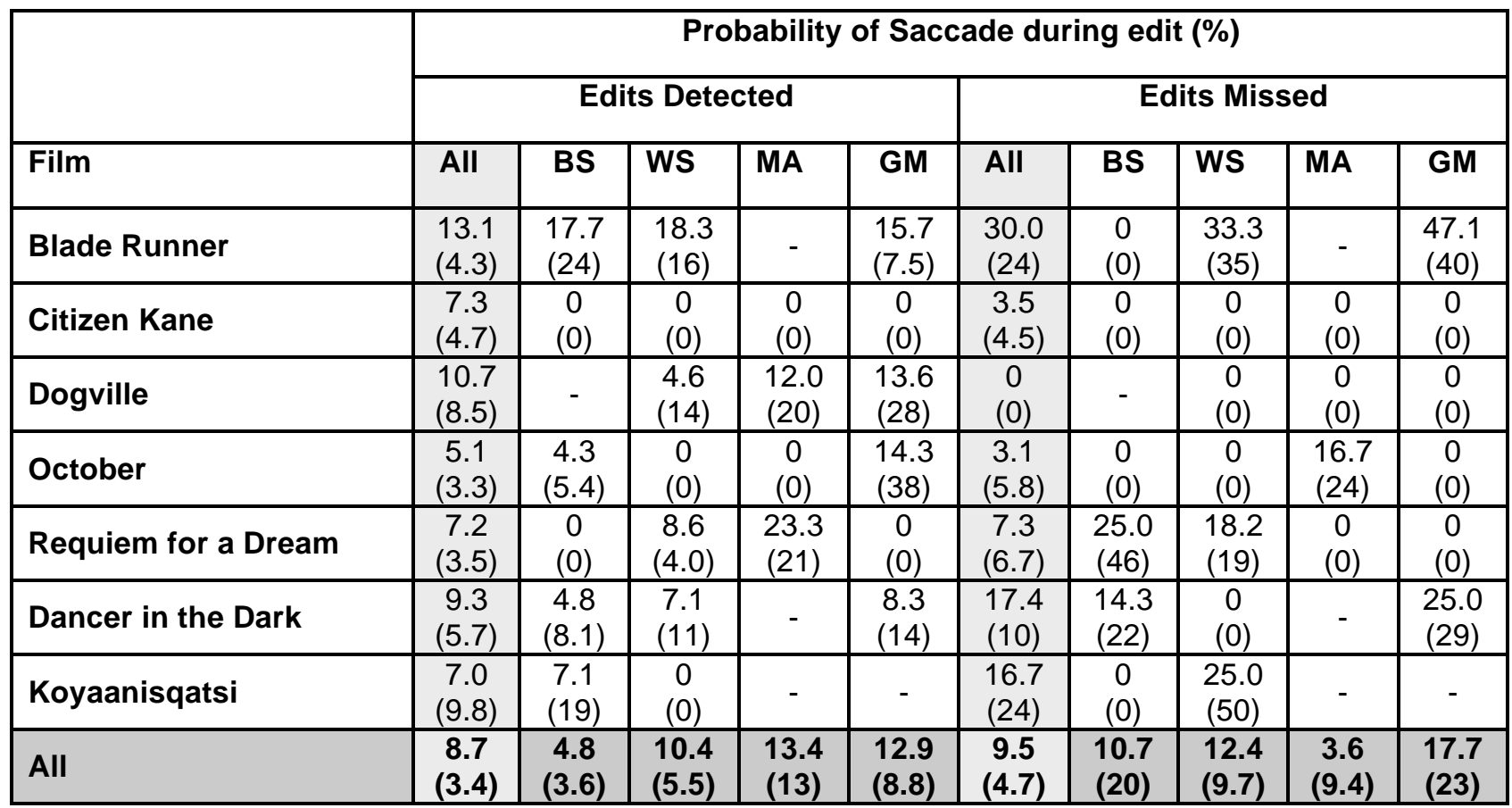

Table 3: Probability (\%) of there being a saccade during an edit which was detected or missed. Results are split by film (rows), presented for all edits (vertical light grey, 'All' columns), for valid cuts with shot size CU, CMS, or MS and type Between Scene (BS), Within Scene (WS), Match Action (MA), and Gaze Match (GM). Means across all films are presented on the bottom row. Standard Deviations are presented in parantheses.

\section{Coincidence of Saccadic Eye Movements and Cuts.}

$8.8 \%$ of all edits coincided with a saccade. Of the edits that were detected, $8.7 \%$ coincided with a saccade. $9.5 \%$ of the missed edits coincided with a saccade. This probability varied considerably across films with some films exhibiting higher probabilities for missed than detected edits while the rest showed similarly low or lower saccade probabilities for missed compared to detected edits (see Table 3). Film editors have not predicted that all edits would coincide with saccades rather that some cuts created according to the Continuity Editing rules, namely Match Action and Gaze Match may create edit blindness by coinciding with saccades. The saccade probability for the four sub-categories of cuts (Table 3, bottom row) tended to be slightly greater for the missed cuts $(11.1 \%)$ than detected cuts $(10.4 \%)$ but a repeated-measures ANOVA revealed no main effect of cut detection $(\mathrm{F}(1,6)=.082, \mathrm{p}=.784$, n.s. $)$ or cut type $(\mathrm{F}(3,18)=.924$, $\mathrm{p}=.449$, n.s. $)$ or interaction $(\mathrm{F}(3,18)=1.033, \mathrm{p}=.401$, n.s.). The overall trend for more missed cuts to coincide with saccades may be evidence of a mechanism whereby awareness of some cuts is limited by saccadic suppression. However, the low number of missed cuts introduced too much variance to verify this effect. In conflict with this trend, Match Action cuts exhibited a significant effect in the other direction: missed cuts were significantly less likely to coincide with a saccade $(\mathrm{t}(6)=2.649, \mathrm{p}<.05)$. This indicates that during the majority of missed Match Action cuts the viewers' eyes were open and fixating, providing no period of perceptual insensitivity.

The saccade probability presented above represents the probability that the eyes were moving at the precise moment a cut occurred. As with eye blinks, the suppression of perceptual sensitivity during a saccade (20-50ms) actually begins about $75 \mathrm{~ms}$ before the eye movement and last around $50 \mathrm{~ms}$ after the eyes have landed (Diamond, Ross, \& Morrone, 2000). A 
repeated-measures ANOVA of Saccade Probability with the factors Time $(100 \mathrm{~ms}$ time bins beginning $300 \mathrm{~ms}$ before the cut and extending $700 \mathrm{~ms}$ after the cut) and Cut Type (see Figure 2) revealed a main effect of Cut Type $(\mathrm{F}(3,18)=6.875, \mathrm{p}<.01)$, Time $(\mathrm{F}(9,54)=2.268, \mathrm{p}<.05)$, and a significant interaction $(\mathrm{F}(27,162)=1.849, \mathrm{p}<.05)$. The main effect of Cut Type can be attributed to Within Scenes and Gaze Match having a greater overall average Saccade Probability compared to Between Scenes (both $\mathrm{p}<.05$ ). The main effect of Time was due to a peak in Saccade Probability 200-300ms after the cut across all Cut Types $(\mathrm{p}<.05)$. The only Cut Types that varied from this pattern were Within Scene and Gaze Match. Within Scene cuts exhibited an earlier (beginning $100 \mathrm{~ms}$ post-cut) and more pronounced peak in Saccade Probability (200-300ms) compared to Between scenes (100ms and 200ms, p<.05) and Match Action (200ms, $\mathrm{p}<.05)$. Gaze Match cuts exhibited a significantly earlier peak in Saccade Probability $100 \mathrm{~ms}$ before the cut ( $\mathrm{p}<.05$ compared with all other Cut Types) and lasting to $300 \mathrm{~ms}$ post-cut. This early peak indicates that significantly more saccades were occurring in anticipation of Gaze Match cuts $(13.6 \%)$ than in any other cut type $(\sim 8 \%)$. This difference in saccade probabilities may indicate that, as predicted by film editors (e.g. Dmytryk, 1986) a proportion of Gaze Match cuts may fail to be detected due to their suppression during a saccadic eye movement.

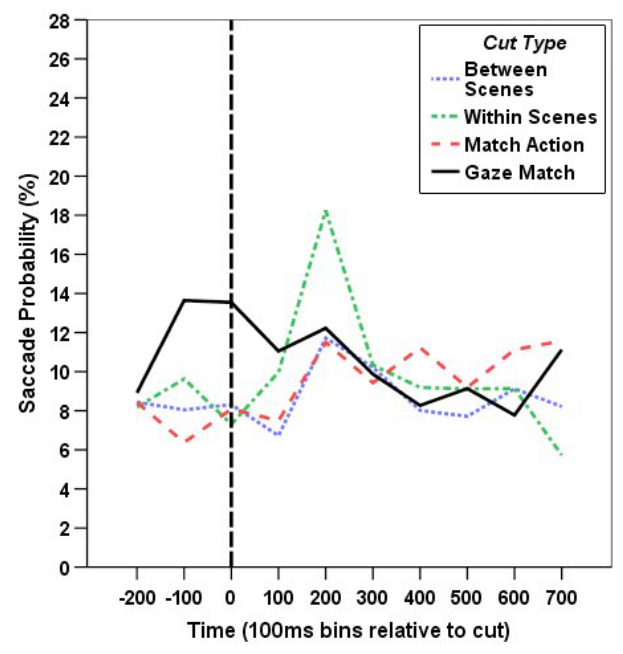

Figure 2: Saccade probability in 100ms bins before and after the cut across the four cut types.

\section{DISCUSSION}

The main hypothesis tested in this investigation was that the application of the Continuity Editing Rules would result in greater edit blindness than edits not composed according to the rules. Overall, the results support this hypothesis. Edits without any continuity of scene, action, or gaze (Between scene) were missed significantly less often $(9.4 \%)$ than edits with continuity of scene (Within Scene=25.1\%) or scene and action (Match Action=32.4\%). The only continuity edits that did not exhibit significantly greater edit blindness were Gaze Match cuts (10.9\%). The percentage of missed Within Scene and Match Action cuts may not seem remarkably high considering that other change blindness experiments report higher rates of change blindness, e.g. $66 \%$ failure to detect change to a centrally attended actor across a cut (Levin \& Simons, 1997). However, participants in these experiments are not aware of the change detection task until after the stimulus has been presented and when they are told to look for the change, a task comparable to our "detect the edit" task, their failure rate drops to $<5 \%$ (Levin \& Simons, 1997). In static scenes, even slight changes to the global luminance or contrast of a scene occurring without any occlusion (e.g. saccade) or distraction (e.g. flicker) are missed $0 \%$ of the time (Henderson, Brockmole, \& Gajewski, 2008). Therefore, compared to existing studies, the 9-32\% failure to detect total, global scene changes (i.e. edits) in this study is remarkable considering that the participants only task was cut detection. This indicates that edit blindness occurs to some degree across all edit types and can be increased by adhering to the continuity editing rules.

In terms of the other measure of edit blindness cut detection times - these were longest for Match Action cuts and shortest for Gaze Match cuts. The cut detection times for all continuity edits were expected to be longer than the no continuity condition (Between Scene) but this is clearly not the case (except a trend in the right direction for Match Action cuts). These quick detection times seem to suggest that for Within Scene and Gaze Match cuts participants either detect or miss a cut, there is never any ambiguity that might lead to longer detection times. By comparison, Match Action cuts may exhibit longer detection times due to the ambiguous timing of the cut: the motion blur caused by 
the sudden onset of motion prior to the cut may obscure the cut itself, increasing the probability of edit blindness and delaying recognition that a cut has occurred until the image has stabilised.

For those cuts that were missed, only Within Scene cuts showed any signs that the visual transients associated with the cuts may have been masked by eye blinks. Most cut types (except Match Action) showed a greater probability of saccadic eye movements during missed compared with detected cuts but these differences were not significant. Only Gaze Match cuts showed any sign of anticipatory saccadic eye movements that may have masked cuts. However, given the low percentage of missed Gaze Match cuts it is unclear whether these anticipatory eye movements resulted in edit blindness. In general, the most reliable method for creating edit blindness appears to be maintaining scene continuity across a cut and coinciding the cut with a sudden onset of visual motion, i.e. creating a Match Action cut.

Why did maintaining scene continuity (Within Scene cuts) result in a significantly greater degree of edit blindness compared with cuts with no continuity (Between Scene cuts)? All cuts used in the main analysis of this study were visually very similar: the shot following the cut had to contain at least one human-like figure framed by the shot from the waist up or closer (CU, CMS, or MS). The only difference between Within Scene and Between Scene cuts was whether the new shot was immediately recognisable as belonging to the same scene as the previous shot. This slight difference appears to have had a significant impact on how participants attended to the new shot and the degree to which they were aware of the cut.

Although the only task in the present study was cut detection, it appears that participants divided their attention between this task and following the film narrative. Allocating attention to this secondary task may have resulted in insufficient attention being made available to the cut detection task. Such an absence of awareness due to failure to attend to a visual feature of a scene is referred to as inattentional blindness (Mack $\&$ Rock, 1998). Studies investigating how we perceive realistic human activities, such as washing the dishes or ironing a shirt, have revealed that we parse observed activities into perceptual units (Newtson, 1973; Zacks et al., 2001). During the boundaries between these units, cognitive resources may be occupied with encoding the perceptual unit in memory, leaving insufficient resources to detect disruptions to the image (Levin \& Varakin, 2004). Within Scene cuts typically coincide with shifts in action that may be perceived as perceptual boundaries (Schwan, Garsoffky, \& Hesse, 2000). Evidence for the modulation of viewer attention during the perception of human events is still speculative (see Smith, 2006, chapter 3 for further discussion) but if it were to be corroborated it may explain why editors choose to cut during changes in action. By choosing to cut during a perceptual boundary, a film editor may be limiting the attentional resources available for detecting a cut while ensuring that the cut itself is not processed as an artificial perceptual boundary. By comparison, a Between Scene cut typically occurs at the end of a scene when all action has ended and the viewer has no expectation of what will happen next. This lack of expectation and the disorientation caused by a Between Scene cut can be seen in the time taken to orient to the new shot. Within Scene cuts exhibit an early peak in saccadic activity beginning 0-100ms following a cut (Figure 2). By comparison, participants took longer to orient to breaks in scene continuity: the peak in saccadic activity for Between Scene cuts begins 100ms after Within Scene cuts $^{6}$. The break in attention experienced after Between Scene cuts may be responsible for the lower incidence of missed cuts: viewers were unable to immediately orient to the new shot allowing them instead to allocate attention to the cut detection task.

Alternatively, Between Scene cuts may actually increase expectation of a cut due to the termination of all predictable action ${ }^{7}$. This would enable viewers to quickly and reliably detect the cut when it occurs but slow their orienting to the new shot due to its

\footnotetext{
${ }^{6}$ A similar peak in saccadic activity $200-400 \mathrm{~ms}$ following film cuts has been reported in previous studies and is thought to indicate saccades initiated in response to the new shot (Hochberg \& Brooks, 1978; d'Ydewalle and Venderbeeken, 1990; d'Ydewalle, Desmet, \& Van Rensbergen, 1998; May, Dean, \& Barnard, 2003; Carmi \& Itti, 2006).

${ }^{7}$ This interpretation was suggested by an anonymous reviewer.
} 
unexpected content and lack of relationship to the previous shot. In this situation viewers may initially orient to visually salient features of the new shot rather than semantically or narratively significant objects (Carmi \& Itti, 2007; also see Nyström \& Holmqvist in this volume for discussion of saliency vs. semantics). Such fresh appraisal of the scene may take longer than orienting to expected features and therefore account for the delayed rise in saccade frequency observed after Between Scene compared with Within Scene cuts. However, a direct comparison of the influence of semantic relatedness and visual saliency on overt attention following cuts would be required to further understand the differences in edit blindness and saccadic activity between Between Scene and Within Scene cuts.

The relationship between the timing of Within Scene cuts and cognitive event boundaries may also explain the higher coincidence of blinks with missed Within Scene cuts. The film editor Walter Murch (2001) hypothesised that blinks function as punctuation to cognitive events, and by identifying these cognitive event boundaries, blinks could be used to hide cuts. Some evidence of a relationship between blink frequency and cognitive processing does exist (Fogarty \& Stern, 1989; Ichikawa \& Ohira, 2004; Fukuda et al., 2005) although attempts to find a direct relationship between blinks and event boundaries have failed (Smith, Whitwell, and Lee, 2006). Further examination of the relationship between blinks and Within Scene cuts is required to establish if the relationship found in the present study is replicable.

Why did the addition of gaze continuity to scene continuity (Gaze Match cuts) result in less edit blindness? Match Action and Gaze Match cuts are visually and conceptually very similar to Within Scene cuts. They all depict on-going scenes containing recognisable human-like figures framed in a similar way. The only difference is that Match Action cuts are preceded by a sudden onset of motion and Gaze Match cuts by a look off-screen. How can these slight differences result in such varying degrees of edit blindness?

Match Action cuts appear to improve upon Within Scene cuts by adding motion blur to scene continuity.
This motion blur seems to function in a similar way to the 'mudsplats' used in change blindness studies (O'Regan et al., 1999): filling the scene with motion transients so that the transients associated with the cut do not capture attention (a similar effect has been reported in attention capture paradigms: Pinto, Olivers, $\&$ Theeuwes, 2008). By comparison, Gaze Match cuts direct viewer attention across the cut using a gaze cue. The higher incidence in saccadic activity before and after Gaze Match cuts indicates that the gaze cue is effective in directing attention (see Figure 2) but this does not result in an increase in edit blindness. Instead what appears to happen is that some of these attentional shifts may coincide with the cut, suppressing sensitivity to the cut transients, but the majority of shifts will probably terminate before or begin after the cut allowing the viewer to perceive the cut transients. The majority of Within Scene cuts seem to overcome the problem of visible cut transients by occupying the viewer with the task of orienting to the new shot. For Gaze Match cuts, no such orienting is required as the gaze cue prior to the cut has already indicated where the centre of interest will be in the new shot. This may mean that greater attentional resources are available for the cut to be processed to the level of awareness if the viewing task requires it. However, under normal viewing conditions the viewer's primary interest should be on following the depicted action, not detecting edits. This may mean that Gaze Match cuts result in quick and direct orienting of attention across the cuts without the cut itself reaching the level of conscious awareness. More subtle methods for gauging viewer awareness of cuts, such as testing implicit memory for the cut (similar to implicit change detection: Levin, Simons, Angelone, \& Chabris, 2002; Hollingworth \& Henderson, 2002; Henderson \& Hollingworth, 2003b; Angelone, Levin, \& Simons, 2003) may be required to examine edit blindness during normal film viewing. The precise timecourse of gaze cuing in dynamic scenes, the reliability of such cues, and detailed analysis of where the viewers eyes go in response to the cue will be investigated in subsequent studies.

What is the implication of edit blindness on our understanding of how we represent dynamic visual scenes? One interpretation of edit blindness 
could be that we retain little or no information about scene content during film viewing and are, therefore unable to detect changes to this information. The results presented here clearly do not support this view. The majority of cuts are detected (70-90\%; the inverse of Table 2, bottom row, $1^{\text {st }}$ block) even when the transients associated with some of those cuts are suppressed during saccadic eye movements ( $\sim 10 \%$, Table 3 , bottom row, $2^{\text {nd }}$ block). Viewers must be retaining some form of visual representation in order for them to compare the new shot to the old and detect the change. However, when edit blindness occurs either A) this comparison is not performed, or B) the representation is too sparse for the comparison to reveal the difference. Studies investigating the representations constructed during static scene viewing have revealed that these representations are richly detailed both at the object and scene level (Henderson \& Hollingworth, 1999; Hollingworth et al., 2001; Melcher, 2001; Hollingworth \& Henderson, 2002; Tatler, Gilchrist, \& Land, 2005; Hollingworth, 2006). However, it is currently unknown if similarly rich representations are retained during dynamic scene viewing. Recognition memory for dynamic scenes has been shown to be better than static scenes (Matthews, Benjamin, \& Osborne, 2007) but the content of this memory does not seem to be used to detect changes (Levin \& Simons, 1997; 2000; Angelone, Levin, \& Simons, 2003). Evidence from developmental studies (Xu \& Carey, 1996), multiple object tracking (Kahneman, Treisman, \& Gibbs, 1992; Scholl, 2001; Mitroff \& Alvarez, 2007), and change blindness (Levin \& Simons, 1997) suggest that changes to dynamic scenes will only be detected if they violate the spatiotemporal continuity of focal objects within the scene. Our results support this view by indicating that edit blindness is more prevalent for cuts that present a continuing action (Within Scene and Match Action). However, assumptions about the spatiotemporal continuity of objects presented in film cannot be the same as the spatiotemporal continuity of objects in the real-world as $3 \mathrm{D}$ space is transformed and contorted to accommodate the constraints of the $2 \mathrm{D}$ screen. What form do these spatiotemporal expectations take during film viewing? How detailed are our representations of object and scene details? How are these representations updated across edits in the absence of explicit awareness of the editing? How do the representations and expectations of continuity during film viewing differ from those constructed during real-world dynamic scene viewing?

All of these questions are currently unanswered and require dedicated empirical investigation if we are to know whether our current theories about how static visual scenes are perceived and represented in memory scale up to film and real-world dynamic scene viewing. For instance, it is hard to believe that we would fail to notice the equivalent of an edit occurring during natural viewing of a real-world scene. If we were instantaneously transported to a different location in the real-world we would expect to notice even if we were attending to the same object before and after the change (equivalent to a Match Action cut) and the change coincided with a saccade or a sudden onset of motion. It could be argued that Edit Blindness is unique to film viewing due to the artificial nature of film and the specialised viewing behaviour we have developed to accommodate the differences between film and reality. However, other change blindness phenomenon initially reported in film (Levin \& Simons, 1997) have been shown to replicate in the realworld (Simons \& Levin, 1998). If the same real-world replication could be demonstrated for edit blindness it would have serious implications for theories about how we represent peripheral information, maintain object representations during dynamic scenes, orient our viewpoint within space, and distribute attention during dynamic scenes.

\section{CONCLUSION}

The study reported here presents the first empirical evidence of edit blindness. Film editors' intuitions about the techniques that minimize viewer awareness of editing are validated in a cut detection task. During the perception of human activity, attention appears to fluctuate, providing moments when visual attention is absent or suppressed during eye blinks and saccadic eye movements. The Continuity Editing rules allow film editors to identify these moments in order to minimise viewer awareness of edits. Understanding precisely how the Continuity Editing rules function and what information is represented and monitored during film viewing will further our understanding of how we experience film and real-world dynamic scenes. 


\section{ACKNOWLEDGEMENTS}

Thanks to Gustav Kuhn for help acquiring data, Julia Schmidt for hand coding the edits, Andrea Greve and Melissa Vo for detailed comments on drafts of this paper and the members of the Edinburgh University Visual Cognition Lab and Eye Movement User group for their feedback during early presentations of this data. Ths research was funded by ESRC grant RES062-23-1092.

\section{REFERENCES}

Anderson, J. (1996). The Reality of Illusion: An Ecological Approach to Cognitive Film Theory. Carbondale, USA: Southern Illinois University Press.

Angelone, B. L., Levin, D. T., \& Simons, D. J. (2003) The relationship between change detection and recognition of centrally attended objects in motion pictures. Perception, 32, 947-962.

Birmingham, E., Bischof, W.F., \& Kingstone, A. (2007). Why do we look at eyes? Journal of Eye Movement Research, 1(1):1, 1-6,

Bordwell, D., \& Thompson, K. (2001) Film Art: An introduction (6th ed.,). New York, USA: Mc Graw Hill.

Bristow, D., Haynes, J.-D., Sylvester, R., Frith, C. D., \& Rees, G. (2005) Blinking Suppresses the Neural Response to Unchanging Retinal Stimulation. Current Biology, 15, 1296-1300.

Brockmole, J. R., \& Henderson, J.M. (2005). Prioritization of new objects in real-world scenes: Evidence from eye movements. Journal of Experimental Psychology: Human Perception and Performance, 31, 857-868.

Burr, D. (2005) Vision: in the Blink of an Eye. Current Biology, 15, R554-R556.

Carlson-Radvansky, L. A., \& Irwin, D. E. (1995) Memory for structural information across eye movements. Journal of Experimental Psychology: Learning, Memory and Cognition. 21, 1441-58.

Carmi, R., \& Itti, L. (2006). The role of memory in guiding attention during natural vision. Journal of Vision, 6(9):4, 898-914
Carroll, J. M., \& Bever, T. G. (1976) Segmentation in Cinema Perception. Science, 191, 1053-1055.

Castelhano, M. S., Wieth, M. S., \& Henderson, J. M. (2007). I see what you see: Eye movements in realworld scenes are affected by perceived direction of gaze. In L. Paletta and E. Rome (Eds.), Attention in Cognitive Systems (pp 252-262). Berlin: Springer.

d'Ydewalle, G., Desmet, G., \& Van Rensbergen, J. (1998) Film Perception: The processing of film cuts. G. Underwood (Ed.), Eye guidance in reading and scene perception (pp. 357-367). Oxford: Elsevier.

d'Ydewalle, G., \& Vanderbeeken, M. (1990). Perceptual and cognitive processing of editing rules in film. R. Groner, G. d'Ydewalle, \& R. Parnham (Eds.), From eye to mind: Information acquisition in perception, search, and reading. (pp. 129-139). Amsterdam: Elsevier (North Holland).

Diamond, M. R., Ross, J., \& Morrone, M. C. (2000) Extraretinal Control of Saccadic Suppression. The Journal of Neuroscience, 20, 3449-3455.

Dmytryk, E. (1986). On Filmmaking. London, UK: Focal Press.

Fogarty, C., \& Stern, J. A. (1989). Eye movements and blinks: their relationship to higher cognitive processes. International Journal of Psychophysiology, 8, 35-42.

Friesen, C. K., \& Kingstone, A. (1998). The eyes have it!: reflexive orienting is triggered by nonpredictive gaze. Psychonomic Bulletin and Review, 5, 490-495.

Frith, U., \& Robson, J. E. (1975). Perceiving the language of films. Perception, 4, 97-103.

Fukuda, K., Stern, J. A., Brown, T. B., \& Russon, M. B. (2005). Cognition, blinks, eye-movements, and pupillary movements during performance of a running memory task. Aviation, Space and Environmental Medical, 76(7 Suppl.), C75-85.

Germeys, F. \& d'Ydewalle, G. (2007) The psychology of film: perceiving beyond the cut. Psychological Research, 71, 458-466.

Grimes, J. (1996). On the failure to detect changes in scenes across saccades. In Perception.Vancouver Studies in Cognitive Science, (Ed. K Akins) 5, 89109. New York: Oxford Univ.Press 
Henderson, J. M. (2003). Human gaze control in realworld scene perception. Trends in Cognitive Sciences, 7, 498-504.

Henderson, J. M., Brockmole, J. R., \& Gajewski, D. A. (2008). Differential detection of global luminance and contrast changes across saccades and flickers during active scene perception. Vision Research, $48,16-29$.

Henderson, J. M., \& Hollingworth, A. (1999). The role of fixation position in detecting scene changes across saccades. Psychoogical. Science. 10, 43843.

Henderson, J. M., \& Hollingworth, A. (2003a). Global transsaccadic change blindness during scene perception. Psychological Science, 14, 493-497.

Henderson, J. M., \& Hollingworth, A. (2003b). Eye movements and visual memory: Detecting changes to saccade targets in scenes. Perception \& Psychophysics, 65, 58-71.

Hochberg, J., \& Brooks, V. (1978). Film Cutting and Visual Momentum. J. W. Senders, D. F. Fisher, \& R. A. Monty (Eds.), Eye Movements and the Higher Psychological Functions (pp. 293-317). Hillsdale, NJ: Lawrence Erlbaum.

Hollingworth, A. (2006). Visual memory for natural scenes: Evidence from change detection and visual search. Visual Cognition, 14, 781-807.

Hollingworth, A., \& Henderson, J. M. (2002). Accurate visual memory for previously attended objects in natural scenes. Journal of Experimental Psychology: Human Perception and Performance, 28, 113-136.

Hollingworth, A., \& Henderson, J. M. (2004). Sustained change blindness to incremental scene rotation: A dissociation between explicit change detection and visual memory. Perception \& Psychophysics, 66, 800-807.

Hollingworth, A., Williams, C. C., \& Henderson, J. M. (2001). To see and remember: Visually specific information is retained in memory from previously attended objects in natural scenes. Psychonomic Bulletin \& Review, 8, 761-768.

Humphrey, K., \& Underwood, G. (2008) Fixation sequences in imagery and in recognition during the processing of pictures in real-world scenes. Journal of Eye Movement Research, 2(2): 3, 1-15.
Ichikawa, N., \& Ohira, H. (2004). Eyeblink activity as an index of cognitive processing: temporal distribution of eyeblinks as an indicator of expectancy in semantic priming. Percept Mot Skills, 98(1), 131-40.

Kahneman, D., Treisman, A., \& Gibbs, B. J. (1992). The reviewing of object files: Object-specific integration of information. Cognitive Psychology, 24, 175-219.

Katz, S. D. (1991). Film Directing Shot by Shot: visualizing from concept to screen. CA, US: Michael Wiese Productions.

Kraft, R. N. (1987). Rules and strategies of visual narratives. Perceptual and Motor Skills, 64, 3-14.

Kuhn, G. \& Tatler, B. W. (2005). Magic and fixation: Now you don't see it, now you do. Perception, 34, 1153-1161

Langton, S. R. H., Watt, R. J., \& Bruce, V. (2000). Do the eyes have it? Cues to the direction of social attention. Trends in Cognitive Sciences, 4, 50-59.

Levin, D. T., \& Simons, D. J. (1997). Failure to detect changes to attended objects in motion pictures. Psychonomic Bulletin and Review, 4, 501-506.

Levin, D. T., \& Simons, D. J. (2000). Perceiving stability in a changing world: Combining shots and integrating views in motion pictures and the real world, Media Psychology, 2, 357-380.

Levin, D. T., Simons, D. J., Angelone, B., \& Chabris, C. F. (2002). Memory for centrally attended changing objects in an incidental real-world change detection paradigm. British Journal of Psychology, 93, 289-302.

Levin, D. T., \& Varakin, D. A. (2004). No pause for a brief disruption: Failures of visual awareness during ongoing events. Consciousness and Cognition, 13, 363-372.

Mack, A., \& Rock, I. (1998). Inattentional Blindness. Cambridge, USA: MIT Press.

Matthews, W. J., Benjamin, C., \& Osborne, C. (2007) Memory for moving and static images. Psychonomic Bulletin \& Review, 14, 989-993.

May, J., Dean, M. P., \& Barnard, P. J. (2003). Using Film Cutting Techniques in Interface Design. Human-Computer Interactions, 18, 325-372. 
Melcher, D. (2001). Persistence of visual memory for scenes - A medium-term memory may help us to keep track of objects during visual tasks. Nature, 412(6845), 401-401.

Messaris, P. (1994). Visual Literacy: Image, Mind, and Reality. Boulder, USA: Westview Press.

Mitroff, S. R., \& Alvarez, G. A. (2007) Space and time, not surface features, guide object persistence. Psychonomic Bulletin \& Review, 14, 1199-1204.

Mitroff, S. R., Simons, D. J., \& Levin, D. T. (2004). Nothing compares two views: Change blindness can occur despite preserved access to the changed information. Perception \& Psychophysics, 66, 1268-1281.

Murch, W. (2001). In The Blink Of An Eye: a perspective on film editing. Los Angeles, USA: Silman-James Press.

Newtson, D. (1973). Attribution and the unit of perception of ongoing behavior. Journal of Personality and Social Psychology, 28, 28-38.

Nyström, M. \& Homlqvist, K. (2008) Measuring Causal Effects in Fixation Selection Using Contrast Manipulated Images. Journal of Eye Movement Research, 2(2): 2, 1-11.

O’Regan, J. K., Deubel, H., Clark, J. J., \& Rensink, R. A. (2000). Picture changes during blinks: looking without seeing and seeing without looking. Visual Cognition, 7, 191-211.

O’Regan, J. K., Rensink, R. A., \& Clark, J. J. (1999). Change blindness as a result of 'mudsplashes.' Nature, 398, 34.

Pepperman, R. D. (2004). The Eye is Quicker: film editing; making a good film better. Los Angeles, USA: Michael Wiese Productions.

Pinto, Y., Olivers, C. L., \& Theeuwes, J. (2008) Selecting from dynamic environments: Attention distinguishes between blinking and moving. Perception \& Psychophysics, 70, 166-178.

Reisz, K., \& Millar, G. (1953). Technique of Film Editing. London, UK: Focal Press.

Rensink, R. A., O’Regan, J. K., Clark, J. J. (1997) To see or not to see: the need for attention to perceive changes in scenes. Psychological Science, 8, 36873.
Rensink, R. A., O’Regan, J. K., Clark, J. J. (2000) On the failure to detect changes in scenes across brief interruptions. Visual Cognition, 7, 127-45.

Scholl, B. J. (2001). Objects and attention: The state of the art. Cognition, 80, 1 - 46.

Schröder, J. (1990). Die psychologische Realität von Prinzipien des Continuity Cinema. G. Schumm, \& H. G. Wulff (Eds.), Film und Psychologie I. Kognition-Rezeption-Perzeption (pp. 109-142). Münster, Germany: Maks Publikationen.

Schwan, S., Garsoffky, B., \& Hesse, F. W. (2000). Do film cuts facilitate the perceptual and cognitive organization of activity sequences? Memory \& Cognition, 28, 214-223.

Simons, D. J. (1996) In sight, out of mind: when object representations fail. Psychological Science, 7, 3015 .

Simons, D. J. (2000). Attentional capture and inattentional blindness. Trends in Cognitive Sciences, 4, 147-155.

Simons, D. J., \& Chabris, C. F. (1999). Gorillas in our midst: sustained inattentional blindness for dynamic events. Perception, 2, 1059-1074.

Simons, D. J., \& Levin, D. T. (1997). Change Blindness. Trends in Cognitive Science, 1, 261-267.

Simons, D. J., \& Levin, D. T. (1998). Failure to detect changes to people during a real-world interaction. Psychonomic Bulletin and Review, 5, 644-49.

Smith, T. J. (2006) An Attentional Theory of Continuity Editing. Doctoral Thesis. University of Edinburgh.

Smith, T. J., Whitwell, M., \& Lee, J. (2006). Eye Movements and Pupil Dilation During Event Perception. Proceedings of the Eye Tracking Research and Applications conference 2006.

Tatler, B. W., Gilchrist, I. D., \& Land, M. F. (2005). Visual memory for objects in natural scenes: From fixations to object files. Quarterly Journal of Experimental Psychology Section A-Human Experimental Psychology, 58(5), 931-960.

Tatler, B. W. \& Kuhn, G. (2007). Don't look now: The magic of misdirection. In R. van Gompel, M. Fischer, W. Murray \& R. Hill (Eds.) Eye Movement Research: Insights into mind and brain. Elsevier, 697-714 
Võ, M. L-H., Schneider, W. X., \& Matthias, E. (2008) Transsaccadic Scene Memory Revisited: A , Theory of Visual Attention (TVA)' Based Approach to Recognition memory and Confidence for Objects in Naturalistic Scenes. Journal of Eye Movement Research, 2(2): 7, 1-13.

Xu, F., \& Carey, S. (1996). Infants' metaphysics: the case of numerical identity. Cognitive Psychology, 30, 111-153.

Yarbus, A. (1967). Eye Movements and Vision. New York: Plenum Press.

Zacks, J., Braver, T. S., Sheridan, M. A., Donaldson, D. I., Snyder, A. Z., Ollinger, J. M., Buckner, R. L., \& Raichle, M. E. (2001). Human brain activity time-locked to perceptual event boundaries. Nature Neuroscience, 4, 651-655. 